\title{
Current Status of Anti-Vascular Endothelial Growth Factor Therapy in Europe
}

\author{
Sebastian Wolf \\ Department for Ophthalmology, Inselspital, University of Bern, Bern, Switzerland
}

\begin{abstract}
Vascular endothelial growth factor (VEGF) is an important modulator of angiogenesis, and has been implicated in the pathology of a number of conditions, including age-related macular degeneration (AMD), diabetic retinopathy, and cancer. AMD is a progressive disease of the macula and the third major cause of blindness worldwide. If not treated appropriately, AMD can progress rapidly, causing legal blindness within months of the second eye becoming affected. Until recently, the treatment options for AMD have been limited, with photodynamic therapy (PDT) the mainstay treatment. Although PDT is effective at slowing disease progression, it rarely results in improved vision. Pegaptanib and ranibizumab are both anti-VEGF therapies licensed for the treatment of neovascular AMD in Europe; however, these drugs are not yet available in Japan. This article reviews the available clinical data on anti-VEGF therapies for the treatment of neovascular AMD in Europe, and considers the future of this exciting therapy. Jpn J Ophthalmol 2008;52:433-439 (C) Japanese Ophthalmological Society 2008
\end{abstract}

Key Words: age-related macular degeneration, pegaptanib, ranibizumab, vascular endothelial growth factor, visual acuity

\section{Introduction}

The vascular endothelial growth factor (VEGF) family of growth factors are important modulators of the angiogenesis process, mediating vascular permeability and the formation of new blood vessels from the existing vasculature. ${ }^{1}$ Discovery of the involvement of VEGF in a number of pathologic conditions, including neovascular age-related macular degeneration (AMD), diabetic retinopathy, and cancer, has prompted the development of several antiVEGF therapies for the treatment of these conditions.

The first anti-VEGF therapy, pegaptanib (Macugen, OSI Pharmaceuticals, Melville, NY, USA, and Pfizer, New York, NY, USA), was approved in Europe in 2006 for the treatment of neovascular AMD, shortly followed by the approval of ranibizumab (Lucentis, Novartis Pharmaceuticals, Basel, Switzerland) for the treatment of neovascular AMD in 2007. As yet, neither of these drugs is available in Japan;

\footnotetext{
Received: June 23, 2008 / Accepted: July 14, 2008

Correspondence and reprint requests to: Sebastian Wolf, Department for Ophthalmology, Inselspital, University of Bern, 3010 Bern, Switzerland

e-mail: sebastian.wolf@insel.ch
}

however, pegaptanib was granted orphan-drug status in 2004, and regulatory filing was made in March 2008. This article discusses the current status of anti-VEGF therapy in Europe for the treatment of neovascular AMD, and considers the future of this exciting therapy area.

\section{Disease Background and Epidemiology}

AMD is a progressive disease of the macula and the third major cause of blindness worldwide. ${ }^{2,3}$ In its late stages, AMD results in the loss of central vision due to photoreceptor dysfunction caused either by geographic atrophy or by choroidal neovascularisation (CNV). Two distinct processes lead to this vision loss: geographic atrophy (atrophic or dry AMD) and CNV (neovascular, exudative, or wet AMD). In atrophic AMD, the choriocapillaries and associated retinal pigment epithelium atrophy, whereas in neovascular AMD, new vessels grow from the choriocapillaries, breaching Bruch's membrane and the retinal pigment epithelium to invade the retina. ${ }^{4}$ These fenestrated and fragile new vessels leak fluid, lipids, and blood, resulting in fibrous scarring. ${ }^{5}$ If left untreated, neovascular AMD can develop quickly enough to cause legal blindness within months of 
the second eye becoming affected. Therefore, early detection, diagnosis, and treatment of $\mathrm{CNV}$ is essential to maximise the preservation of central vision, minimise the risk of blindness, and maintain patients' quality of life. ${ }^{6}$

\section{Prevalence}

The Rotterdam Study assessed the prevalence of agerelated maculopathy in an elderly population in the Netherlands. ${ }^{7}$ Prevalence of neovascular AMD was $1.1 \%$ overall $(0.8 \%$ in men and $1.2 \%$ in women), whereas prevalence of atrophic AMD was $0.6 \%$ (equally prevalent in men and women). Prevalence of AMD increased with age, rising to $11 \%$ in people aged 85 years or older. ${ }^{7}$ As the population of Europe ages, an increase in the number of patients with AMD can be expected. A recent Swiss study has estimated that there will be a 2.5-fold increase in the number of late AMD cases in Switzerland by 2050, with numbers rising from 37200 cases in 2005 to 52500 cases in 2020 and 93200 cases in $2050 .{ }^{8}$

\section{Treatment Options for AMD}

Until the recent introduction of anti-VEGF therapy, treatment options for neovascular AMD were limited. In the 1980s, the Macular Photocoagulation Study Group reported that laser treatment of extrafoveal choroidal neovascular lesions was beneficial in delaying loss of vision for at least 5 years. ${ }^{9,10}$ The introduction of photodynamic therapy (PDT) with verteporfin in Europe in 2000 and Japan in 2004 further expanded treatment options for AMD. The Treatment of Age-Related Macular Degeneration with Photodynamic Therapy Study (TAP) reported that after 2 years of treatment, $53 \%$ of patients treated with verteporfin plus PDT lost fewer than 15 letters, compared with $38 \%$ of patients treated with placebo plus PDT. Subgroup analyses revealed that the benefits of PDT with verteporfin were concentrated primarily in patients with classic $\mathrm{CNV}$. $^{11}$

\section{Anti-VEGF Treatment}

The exact mechanisms of the pathogenesis of neovascular AMD are not fully understood; however, increased expression of VEGF in the optic nerve and retina is known to precede retinal neovascularisation. ${ }^{12}$ VEGF mRNA levels have been shown to be upregulated in both clinical and experimental studies of $\mathrm{CNV}^{13-15}$ and the introduction of VEGF to healthy eyes mimics the neovascularisation that is observed during the disease process. ${ }^{16-18}$

\section{Pegaptanib (Macugen)}

Pegaptanib is a specific nucleic acid ligand (aptamer) that binds to VEGF165, the most abundant of the human VEGF isoforms, and thereby inhibits interaction with its receptors. Pegaptanib is licensed in Europe for the treatment of neovascular AMD and is administered by intravitreal injection every 6 weeks for at least 2 years. ${ }^{19}$

The VEGF Inhibition Study in Ocular Neovascularization (VISION) clinical trials investigated the safety and efficacy of pegaptanib for the treatment of neovascular AMD in 1190 patients over 2 years. ${ }^{20-22}$ One trial enrolled patients from the United States and Canada, and the other trial enrolled patients from Europe, Israel, Australia, and South America. At baseline, patients were randomised to one of four treatment groups $(0.3,1$, or $3 \mathrm{mg}$ pegaptanib, or sham), with treatment administered every 6 weeks for 54 weeks (a total of nine treatments). Thereafter, patients receiving pegaptanib were randomised to either continue treatment for a second year or cease treatment. To minimise the number of patients receiving sham treatment for 2 years, at year 1 the sham group was randomised to continuing sham injections, no treatment, or one of the three pegaptanib doses. The primary efficacy endpoint was the proportion of patients losing fewer than 15 letters of visual acuity at week 54 . The aims of the second year of study were to assess the safety of the 2-year treatment with pegaptanib, assess the relative benefit of 2-year treatment over 2 years of usual care, and determine whether a second year of treatment resulted in additional benefits beyond the first year.

In a combined analysis of the data from both trials, all three doses of pegaptanib were efficacious in preventing loss of vision compared with sham injections across all CNV subtypes $;{ }^{20} 70 \%, 71 \%$, and $65 \%$ of patients receiving $0.3,1$, and $3 \mathrm{mg}$ pegaptanib, respectively, experienced a loss of fewer than 15 letters of visual acuity at week 54 , compared with $55 \%$ of patients receiving sham injections $(P<0.001$, $P<0.001$, and $P=0.03$, respectively). In addition, $33 \%$, $37 \%$, and $31 \%$ of patients receiving $0.3,1$, and $3 \mathrm{mg}$ pegaptanib, respectively, maintained their vision or gained vision, compared with $23 \%$ of patients receiving sham injections $(P=0.003, P<0.001$, and $P=0.02$, respectively $) .^{20}$ Figure 1 shows the mean change in visual acuity from baseline to week 54.

Analysis of the 2-year VISION trial data revealed that mean visual acuity was maintained in patients receiving $0.3 \mathrm{mg}$ pegaptanib, and the proportion of patients losing fewer than 15 letters of visual acuity from week 54 to week 102 was half that $(7 \%)$ of those who were assigned to 2 years of sham injections or randomised to stop treatment after 1 year $(14 \%){ }^{21}$ In patients who had a visual acuity of 20/200 or better at baseline, those who were treated with continuous $0.3 \mathrm{mg}$ pegaptanib were less likely to progress to legal blindness in year 2;34\% of patients at week 54 and $35 \%$ of patients at week 102 in this group had a visual acuity of $20 / 200$ or worse in the study eye. In contrast, $24 \%$ of patients assigned to discontinue treatment with pegaptanib at week 54 , and $47 \%$ of patients in the sham injection group, had a visual acuity of 20/200 or worse at week 54, increasing to $38 \%$ and $55 \%$, respectively, at week $102 .^{21}$

Endophthalmitis $(1.3 \%)$, traumatic injury to the lens $(0.7 \%)$, and retinal detachment $(0.6 \%)$ were the most 


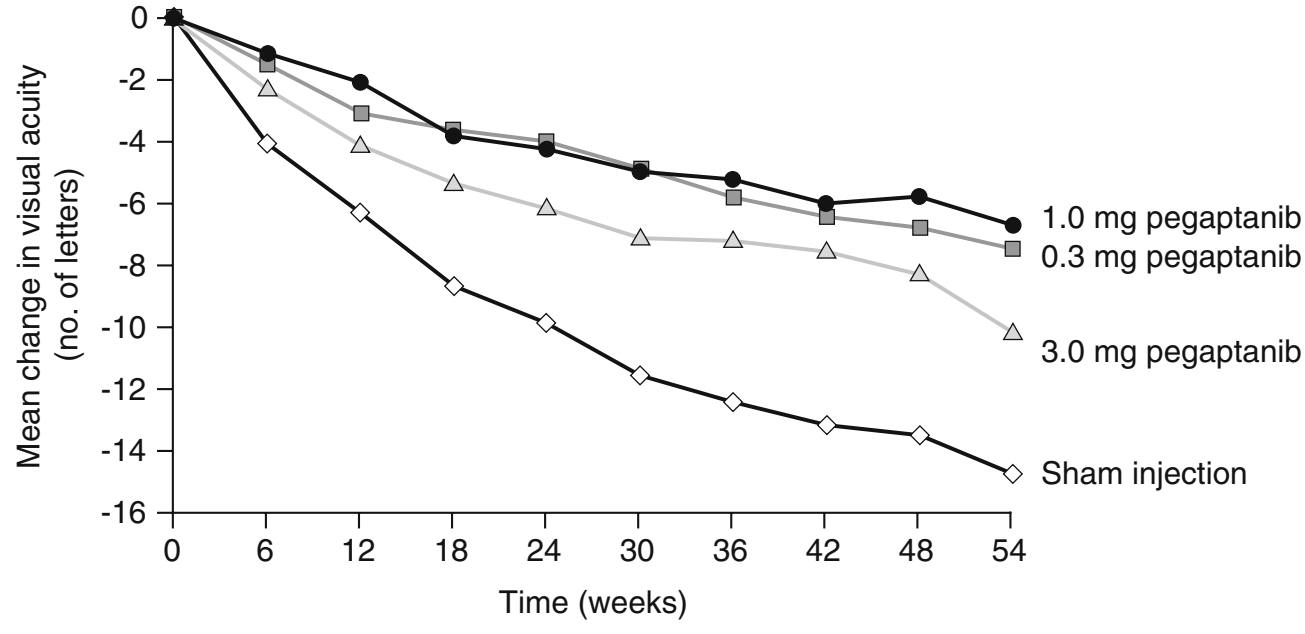

Figure 1. Combined mean change in visual acuity from baseline to week 54 in the VISION clinical trials $(P<0.002$ at every point for the comparison of 0.3 or $1.0 \mathrm{mg}$ pegaptanib with sham injection at week 54, and $P<0.05$ at every point for the comparison of $3.0 \mathrm{mg}$ pegaptanib with sham injection at all other points after baseline).$^{20}$ From ref. 20, with permission. Copyright (C) 2004 Massachusetts Medical Society. All rights reserved. serious injection-related adverse events (AEs) reported in the first year, with two-thirds of cases of endophthalmitis following protocol violations. The authors concluded that these rates were consistent with data in the literature for intravitreous injections and, therefore, the risks associated with the intraocular injection of pegaptanib were probably no worse than those associated with other intraocular treatments. ${ }^{20}$ However, a protocol amendment resulted in no reports of endophthalmitis or traumatic injury to the lens in year $2 .{ }^{22}$ The 2-year results also showed that there was no evidence of an increase in AEs associated with systemic VEGF inhibition, such as hypertension, thromboembolic events, or serious haemorrhagic events. ${ }^{22}$ An additional 1year systemic safety study has confirmed that there is no evidence of systemic VEGF inhibition even at doses up to tenfold the approved $0.3 \mathrm{mg}^{23}$

\section{Ranibizumab (Lucentis)}

Ranibizumab is a humanised monoclonal antibody Fab fragment that inhibits all five human isoforms of VEGF-A, and was specifically designed for the treatment of neovascular AMD. ${ }^{24}$ The MARINA (Minimally Classic/Occult Trial of the Anti-VEGF Antibody to Ranibizumab in the Treatment of Neovascular Age-Related Macular Degeneration) study investigated ranibizumab for the treatment of minimally classic or occult with no classic CNV associated with AMD at 96 sites in the United States. ${ }^{25}$ A total of 716 patients were randomised equally to receive 24 monthly intravitreal injections of 0.3 or $0.5 \mathrm{mg}$ ranibizumab, or sham injections. The primary efficacy endpoint was the proportion of patients losing fewer than 15 letters from baseline visual acuity at 12 months.

At 12 months, $94.5 \%$ of patients treated with $0.3 \mathrm{mg}$ ranibizumab and $94.6 \%$ of those treated with $0.5 \mathrm{mg}$ ranibizumab lost fewer than 15 letters from baseline visual acuity, compared with $62.2 \%$ in the sham injection group $(P<0.001$ for the comparison of each dose with the sham injection group). At 24 months, $92.0 \%, 90.0 \%$, and $52.9 \%$ of patients in the $0.3 \mathrm{mg}$ ranibizumab, $0.5 \mathrm{mg}$ ranibizumab, and sham injection group, respectively, had lost fewer than 15 letters of visual acuity $(P<0.001$ for the comparison of each dose with the sham injection group). Furthermore, at both 12 and 24 months, approximately one-quarter of patients receiving $0.3 \mathrm{mg}$ ranibizumab, and one-third of patients receiving $0.5 \mathrm{mg}$ ranibizumab, had gained 15 or more letters in visual acuity, compared with $5.0 \%$ in the sham injection group at 12 months and $3.8 \%$ at 24 months. Figure $2 \mathrm{~A}$ shows the mean change in visual acuity from baseline to month 24 in this trial. Only $0.8 \%$ of patients receiving $0.3 \mathrm{mg}$ ranibizumab, and $1.2 \%$ of patients receiving $0.5 \mathrm{mg}$ ranibizumab, had severe vision loss (lost 30 letters or more of visual acuity), compared with $14.3 \%$ of patients in the sham injection group. ${ }^{25}$

There was no significant difference between the three treatment groups in the rates of nonocular AEs. In terms of systemic anti-VEGF effects, the rates of hypertension and arterial thrombolic events were similar across groups. Nonocular haemorrhages were similar across all treatment groups in the first year, but cumulative rates increased in all groups in the second year. At 24 months, nonocular haemorrhage rates were $9.2 \%, 8.8 \%$, and $5.5 \%$ in the $0.3 \mathrm{mg}$ ranibizumab, $0.5 \mathrm{mg}$ ranibizumab, and sham injection groups, respectively; these differences were not significant. Over the course of the study, five patients who received ranibizumab experienced presumed endophthalmitis, and six patients receiving ranibizumab experienced serious uveitis. $^{25}$

A second phase III trial (ANCHOR-Anti-VEGF Antibody for the Treatment of Predominantly Classic Choroidal Neovascularisation) compared ranibizumab with verteporfin PDT for the treatment of predominantly classic neovascular AMD. ${ }^{26}$ In this study, 423 patients were randomised equally to receive monthly intravitreal injections of $0.3 \mathrm{mg}$ ranibizumab or $0.5 \mathrm{mg}$ ranibizumab plus sham verteporfin therapy, or monthly sham injections plus active verteporfin therapy. The primary endpoint was the proportion of patients losing fewer than 15 letters from baseline visual acuity at 12 months. The results of this study support the 
A) MARINA

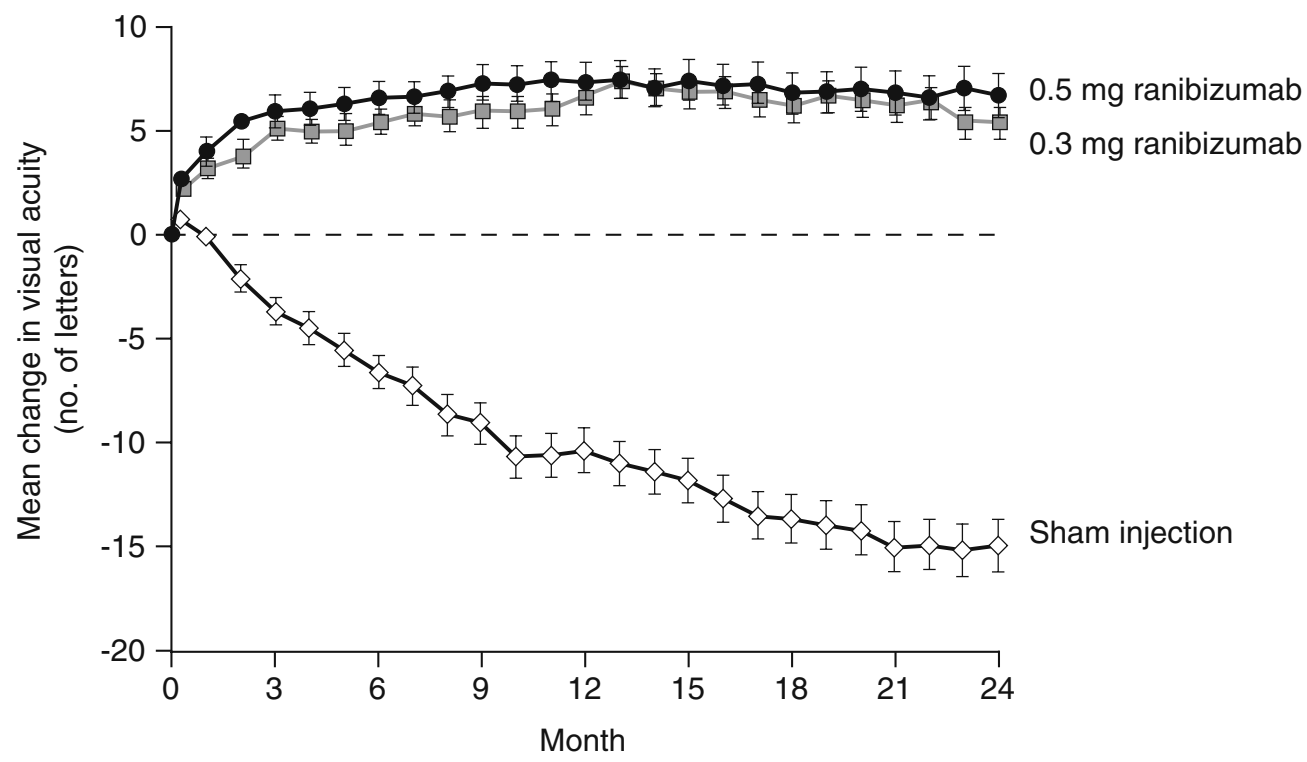

B) ANCHOR

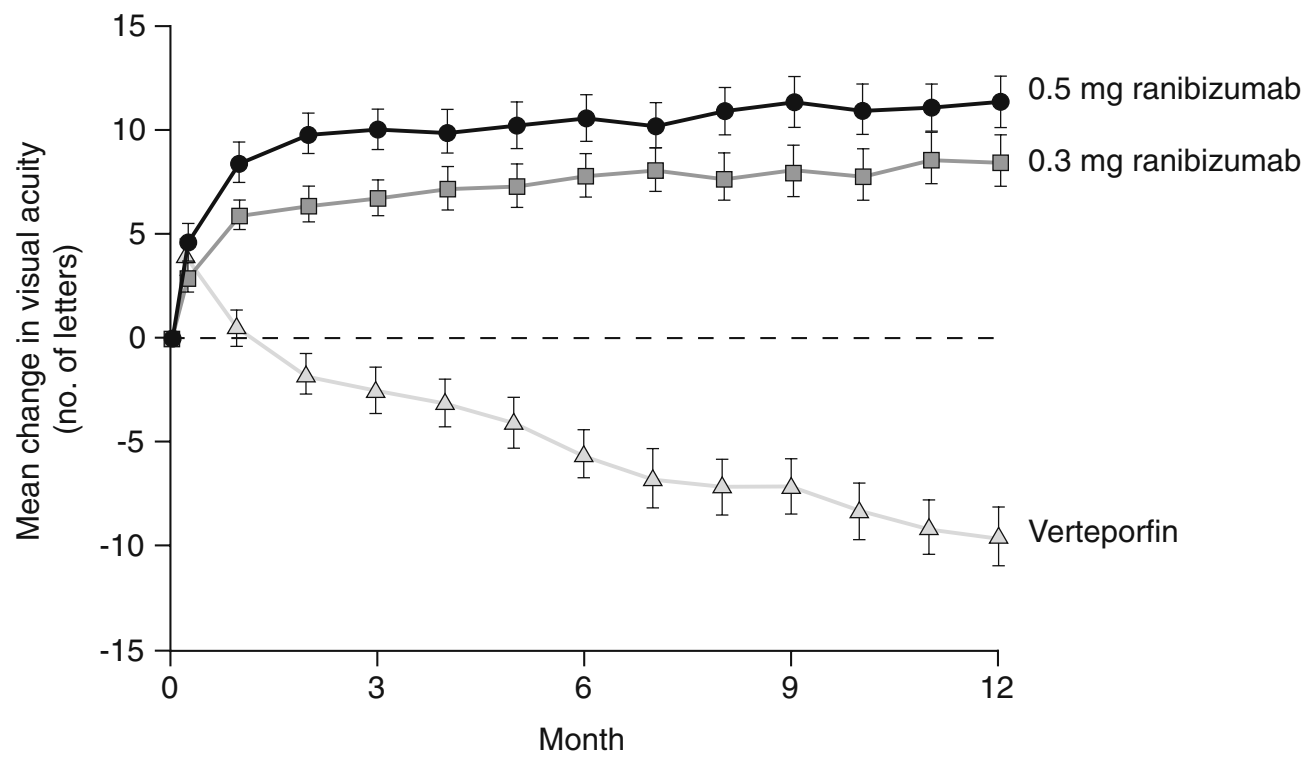

Figure 2A, B. Mean change from baseline in visual acuity from baseline to month 24 in the MARINA (A) and ANCHOR (B) clinical trials. A At each monthly assessment, $P<0.001$ for the comparison between each ranibizumab group and the sham injection group. On day 7 , $P=0.006$ for patients receiving $0.3 \mathrm{mg}$ ranibizumab and $P=0.003$ for patients receiving $0.5 \mathrm{mg}$ ranibizumab. ${ }^{25} \mathbf{B} P<0.001$ for all monthly comparisons of each dose of ranibizumab with verteporfin photodynamic therapy. ${ }^{26}$ From ref. 26, with permission. Copyright (c) 2006 Massachusetts Medical Society. All rights reserved.

findings of MARINA, with $94.3 \%$ of patients receiving $0.3 \mathrm{mg}$ ranibizumab and $96.4 \%$ of patients receiving $0.5 \mathrm{mg}$ ranibizumab losing fewer than 15 letters, compared with $64.3 \%$ of patients receiving verteporfin $(P<0.001$ for each comparison). Visual acuity improved by at least 15 letters in $35.7 \%$ and $40.3 \%$ of patients in the $0.3 \mathrm{mg}$ ranibizumab and $0.5 \mathrm{mg}$ ranibizumab treatment groups, respectively, compared with $5.6 \%$ of patients in the verteporfin treatment group $(P<0.001$ for each comparison). Figure 2B shows the mean change in visual acuity from baseline to month 24 in this trial. Presumed endophthalmitis occurred in two patients $(1.4 \%)$ and serious uveitis in one patient $(0.7 \%)^{26}$
The PIER study evaluated ranibizumab administered monthly for 3 months, followed by quarterly injections. ${ }^{27}$ Using this schedule, ranibizumab provided a significant visual acuity benefit compared with sham treatment; however, treatment was less effective than the monthly schedule employed in MARINA and ANCHOR. ${ }^{27}$ Visual acuity improvements were observed during the first 3 months of the study; however, it then declined during the rest of the 24-month duration of the trial. A subgroup analysis demonstrated that although $40 \%$ of patients retained their initial visual acuity gain during the maintenance phase, the quarterly treatment schedule was not suitable for $60 \%$ of patients. Therefore, more frequent monitoring and 
dosing may be necessary to identify when treatment is needed. To address this question, a second phase IIIb study (SUSTAIN) is currently evaluating the efficacy of 0.3 or $0.5 \mathrm{mg}$ ranibizumab administered monthly for 3 consecutive months (loading phase), followed by monthly retreatment as required for 9 months. ${ }^{28}$ Further doses are administered if visual acuity decreases by five letters or more, or central retinal thickness (CRT) increases by $100 \mu \mathrm{m}$ or more. Treatment was not given if visual acuity was 79 letters or more, or CRT was less than or equal to $225 \mu \mathrm{m}$. An interim analysis of 69 ranibizumab-naïve patients who had completed their 12-month visit showed a mean increase from baseline of 6.8 letters and a mean decrease in CRT of $74.4 \mu \mathrm{m}$ at month 12 , with patients receiving a mean of 5.3 injections (including the three loading-phase injections). Importantly, these improvements were stable from month 3 . Therefore, although these results are only preliminary, it appears that good efficacy outcomes can be achieved with ranibizumab with a lower monthly average frequency of treatment. ${ }^{28}$

\section{Bevacizumab}

Bevacizumab is a humanised monoclonal antibody to VEGF, designed for intravenous administration and currently approved in Europe for the treatment of colorectal cancer and advanced or metastatic renal cell cancer. Bevacizumab is derived from the same murine antibody as ranibizumab; however, it has a different structure and formulation and is not licensed for the treatment of AMD. Bevacizumab has been used on an off-label basis for the treatment of neovascular AMD ${ }^{29,30}$ however, as no randomised clinical trials have been conducted, there is little firm evidence of either its safety or its efficacy in this patient population. In the United States, the National Eye Institute and the National Institute of Health are collaborating on a multicentre clinical trial to compare the safety and efficacy of ranibizumab and bevacizumab for the treatment of neovascular AMD. Likewise, in the UK, the National Health Service is running a similar trial. Results from these trials are expected in the next few years.

\section{Switzerland}

On 30 August 2006, Switzerland became the first European country to approve Lucentis for the treatment of neovascular AMD. Although Lucentis has been fully reimbursed for the treatment of neovascular AMD in Switzerland since the beginning of 2007, treatment with Lucentis is limited to specialised centres and requires data monitoring for a national registry. The official recommendations are to administer Lucentis by intravitreal injection once a month. If monthly injections are not feasible, treatment may be reduced to one injection every 3 months after the first four injections, although this is not as effective.

Currently, it is estimated that Lucentis is used in Switzerland in around $95 \%$ of patients receiving intravitreal

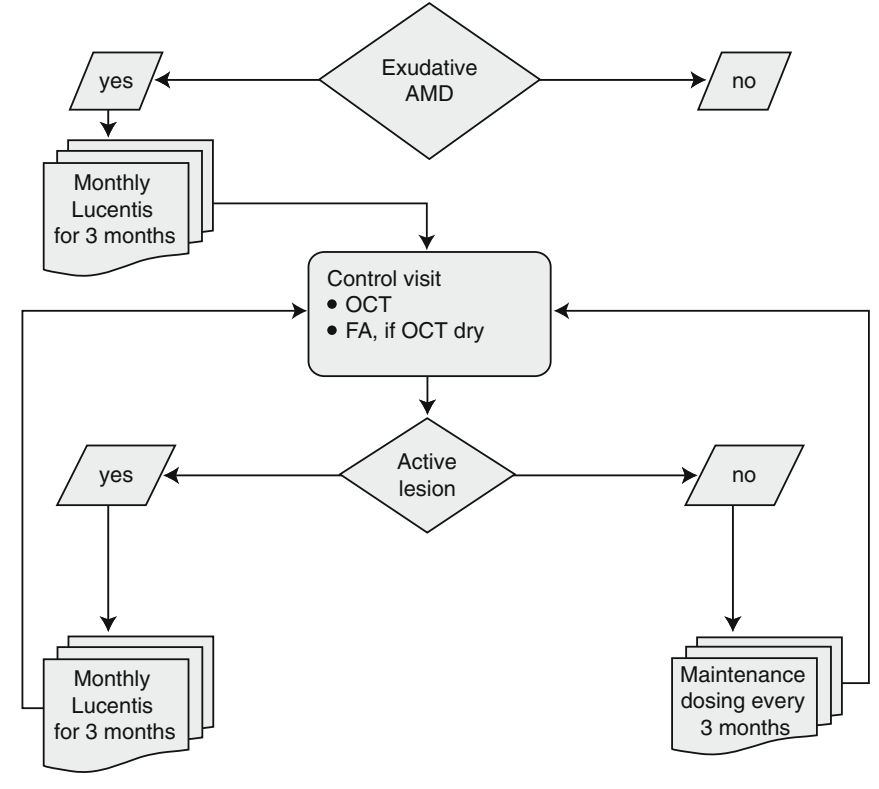

Figure 3. Our current approach for treating patients with neovascular age-related macular degeneration $(A M D)$ with Lucentis (ranibizumab). $O C T$, optical coherence tomography; $F A$, fluorescein angiography.

anti-VEGF treatment for neovascular AMD. In 2007, about 4000 patients with neovascular AMD received 12560 intravitreal Lucentis injections. Treatment-naïve patients received on average 3.99 injections during the first year.

In our centre, we have performed around 2800 Lucentis injections since we began using it for the treatment of neovascular AMD in March 2005. In the beginning, our treatment regimen included three monthly injections followed by dosing as required. Retreatment was initiated if visual acuity deteriorated or lesion activity increased, as shown by either optical coherence tomography (OCT) or by fluorescein angiography; however, using this regimen we were not able to reproduce the exciting results of the ANCHOR or MARINA studies. Therefore, we are currently using a more aggressive approach to treatment (Fig. 3): patients initially receive three monthly intravitreal injections of Lucentis, followed by maintenance dosage every 3 months in patients without active lesions and reinitiation of three monthly injections in patients with active lesions at follow-up visits. An active lesion is defined as new intra- or subretinal haemorrhages, intra- or subretinal fluid accumulation on highresolution OCT, or leakage on fluorescein angiography (Fig. 4). First analysis confirms that this approach may lead to a better visual outcome than the previously used regimen.

\section{Future of Anti-VEGF Therapy}

\section{Anti-VEGF Therapy in Combination with PDT}

Both ranibizumab and pegaptanib are currently being investigated in combination with PDT, with the aim of 

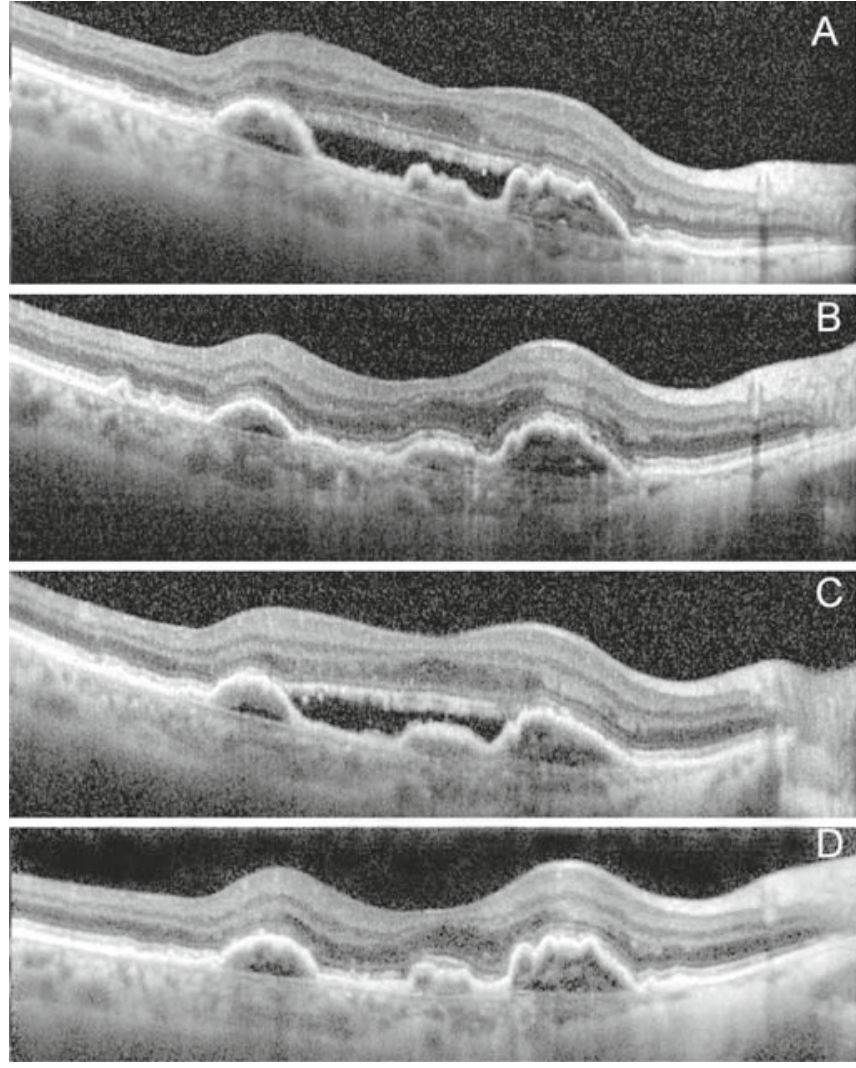

Figure 4A-D. Patient with fibrovascular pigment epithelium detachment. A Initial high resolution OCT (Spectralis HRA+OCT, Heidelberg Engineering, Heidelberg, Germany) showing subretinal fluid accumulation. B High-resolution OCT 4 weeks after three initial intravitreal Lucentis injections showing no subretinal fluid. C New subretinal fluid 3 months after initial Lucentis injections, resulting in initiation of three additional monthly Lucentis injections. D No subretinal fluid 3 months after the second block of Lucentis injections.

potentially reducing the number of intravitreal injections required. A small open-label study of seven patients treated with PDT followed by intravitreal pegaptanib $48 \mathrm{~h}$ later has recently been reported. ${ }^{31}$ In this study, retreatment was indicated with pegaptanib every 6 weeks and with PDT every 12 weeks if fluorescein angiography showed leakage or OCT detected intraretinal oedema. At 24 weeks, patients had an insignificant decrease in mean best-corrected visual acuity from 60.4 letters at baseline to 55.2 letters. The greatest linear dimension increased from 1280 to $2066 \mathrm{~mm}$ $(P=0.043)$, and the total area of $\mathrm{CNV}$ increased from 1.4 to $2.7 \mathrm{~mm}^{2}(P=0.05)$. Although these results are generally poor, the authors suggested that larger-scale controlled studies are required to ascertain whether PDT can reduce the number of intravitreal injections, and which antiangiogenic agent would provide the most potent combination with PDT. ${ }^{31}$

Ranibizumab has also been investigated in combination with PDT. The FOCUS study was a 2-year multicentre, randomised, single-masked, controlled study to examine the safety and efficacy of ranibizumab in combination with verteporfin PDT in 162 patients with neovascular AMD. ${ }^{32}$ Patients received monthly intravitreal ranibizumab or sham injections; all patients also received PDT on day 0 , then quarterly as required. At month $24,88 \%$ of patients receiving ranibizumab plus PDT had lost fewer than 15 letters of visual acuity, compared with $75 \%$ of patients receiving PDT alone $(P<0.05)$. In addition, $25 \%$ of patients in the combination therapy group had gained at least 15 letters, compared with $7 \%$ of patients treated with PDT alone $(P<0.05)$. Overall, patients receiving combination therapy showed less lesion growth and a larger reduction in CNV leakage and fluid accumulation and required fewer PDT treatments than patients treated with PDT alone. The combination treatment group had higher rates of endophthalmitis $(2.4 \%)$ and serious intraocular inflammation $(12.4 \%)$ than patients who received PDT alone ( $0 \%$ for each AE). The incidence of serious nonocular AEs was similar between the two groups. ${ }^{32}$

In conclusion, the development of anti-VEGF therapies has revolutionised the treatment of AMD. The vision improvements associated with these therapies mean that blindness is no longer an inevitable consequence of this condition. Going forward, the combination of anti-VEGF therapies with PDT offers the potential for decreased frequency of injections or further improvement in treatment efficacy. Furthermore, clinical trials of these agents in diabetic retinopathy and macular oedema (secondary to central and branch retinal vein occlusion, and diabetes mellitus) are also currently underway, suggesting that anti-VEGF therapies may have the potential to treat other debilitating eye conditions.

Acknowledgments. The author would like to thank Sarah Feaver, Ph.D., of Complete Medical Communications, who provided editorial support funded by Novartis Pharma AG.

\section{References}

1. Ferrara N, Gerber HP, LeCouter J. The biology of VEGF and its receptors. Nat Med 2003;9:669-676.

2. Kahn HA, Leibowitz HM, Ganley JP, et al. The Framingham Eye Study. I. Outline and major prevalence findings. Am J Epidemiol 1977;106:17-32.

3. Klaver CC, Wolfs RC, Vingerling JR, Hofman A, de Jong PT. Age-specific prevalence and causes of blindness and visual impairment in an older population: the Rotterdam Study. Arch Ophthalmol 1998;116:653-658.

4. de Jong PT. Age-related macular degeneration. N Engl J Med 2006;355:1474-1485.

5. Wong T, Chakravarthy U, Klein R, et al. The natural history and prognosis of neovascular age-related macular degeneration: a systematic review of the literature and meta-analysis. Ophthalmology 2008;115:116-126.

6. Sickenberg M. Early detection, diagnosis and management of choroidal neovascularization in age-related macular degeneration: the role of ophthalmologists. Ophthalmologica 2001;215:247-253.

7. Vingerling JR, Dielemans I, Hofman A, et al. The prevalence of age-related maculopathy in the Rotterdam Study. Ophthalmology 1995;102:205-210.

8. Bauer P, Barthelmes D, Kurz M, Fleischhauer JC, Sutter FK. The potential effect of population development, smoking and 
antioxidant supplementation on the future epidemiology of agerelated macular degeneration in Switzerland. Klin Monatsbl Augenheilkd 2008;225:376-379.

9. Argon laser photocoagulation for neovascular maculopathy. Fiveyear results from randomized clinical trials. Macular Photocoagulation Study Group. Arch Ophthalmol 1991;109:1109-1114.

10. Laser photocoagulation of subfoveal neovascular lesions in agerelated macular degeneration. Results of a randomized clinical trial. Macular Photocoagulation Study Group. Arch Ophthalmol 1991;109:1220-1231.

11. Bressler NM. Photodynamic therapy of subfoveal choroidal neovascularization in age-related macular degeneration with verteporfin: two-year results of 2 randomized clinical trials-tap report 2. Arch Ophthalmol 2001;119:198-207.

12. Amin RH, Frank RN, Kennedy A, Eliott D, Puklin JE, Abrams GW. Vascular endothelial growth factor is present in glial cells of the retina and optic nerve of human subjects with nonproliferative diabetic retinopathy. Invest Ophthalmol Vis Sci 1997;38:36-47.

13. Kvanta A, Algvere PV, Berglin L, Seregard S. Subfoveal fibrovascular membranes in age-related macular degeneration express vascular endothelial growth factor. Invest Ophthalmol Vis Sci 1996; 37:1929-1934.

14. Lopez PF, Sippy BD, Lambert HM, Thach AB, Hinton DR Transdifferentiated retinal pigment epithelial cells are immunoreactive for vascular endothelial growth factor in surgically excised age-related macular degeneration-related choroidal neovascular membranes. Invest Ophthalmol Vis Sci 1996;37:855-868.

15. Yi X, Ogata N, Komada M, et al. Vascular endothelial growth factor expression in choroidal neovascularization in rats. Graefes Arch Clin Exp Ophthalmol 1997;235:313-319.

16. Tolentino MJ, McLeod DS, Taomoto M, Otsuji T, Adamis AP, Lutty GA. Pathologic features of vascular endothelial growth factor-induced retinopathy in the nonhuman primate. Am J Ophthalmol 2002;133:373-385.

17. Tolentino MJ, Miller JW, Gragoudas ES, et al. Intravitreous injections of vascular endothelial growth factor produce retinal ischemia and microangiopathy in an adult primate. Ophthalmology 1996 103:1820-1828

18. Tolentino MJ, Miller JW, Gragoudas ES, Chatzistefanou K, Ferrara N, Adamis AP. Vascular endothelial growth factor is sufficient to produce iris neovascularization and neovascular glaucoma in a nonhuman primate. Arch Ophthalmol 1996;114: 964-970.

19. (OSI) Eyetech Inc and Pfizer Inc. Macugen (pegaptanib sodium injection) prescribing information. 2006.

20. Gragoudas ES, Adamis AP, Cunningham ET Jr, Feinsod M, Guyer DR. Pegaptanib for neovascular age-related macular degeneration. N Engl J Med 2004;351:2805-2816.
21. Chakravarthy U, Adamis AP, Cunningham ET Jr, et al. Year 2 efficacy results of 2 randomized controlled clinical trials of pegaptanib for neovascular age-related macular degeneration. Ophthalmology 2006;113:1508-1525.

22. D'Amico DJ, Masonson HN, Patel M, et al. Pegaptanib sodium for neovascular age-related macular degeneration: two-year safety results of the two prospective, multicenter, controlled clinical trials. Ophthalmology 2006;113:992-1001.

23. Apte RS, Modi M, Masonson H, Patel M, Whitfield L, Adamis AP. Pegaptanib 1-year systemic safety results from a safety-pharmacokinetic trial in patients with neovascular age-related macular degeneration. Ophthalmology 2007;114:1702-1712.

24. Ferrara N, Damico L, Shams N, Lowman H, Kim R. Development of ranibizumab, an anti-vascular endothelial growth factor antigen binding fragment, as therapy for neovascular age-related macular degeneration. Retina 2006;26:859-870.

25. Rosenfeld PJ, Brown DM, Heier JS, et al. Ranibizumab for neovascular age-related macular degeneration. N Engl J Med 2006; 355:1419-1431.

26. Brown DM, Kaiser PK, Michels M, et al. Ranibizumab versus verteporfin for neovascular age-related macular degeneration. $\mathrm{N}$ Engl J Med 2006;355:1432-1444.

27. Regillo CD, Brown DM, Abraham P, et al. Randomized, doublemasked, sham-controlled trial of ranibizumab for neovascular age-related macular degeneration: PIER Study Year 1. Am J Ophthalmol 2008;145:239-248.

28. Meyer CH, Eter N, Holz FG; SUSTAIN study group. Ranibizumab in patients with subfoveal choroidal neovascularization secondary to age-related macular degeneration. Interim results from the SUSTAIN trial. Invest Ophthalmol Vis Sci 2008;49 (in press).

29. Moshfeghi AA, Rosenfeld PJ, Puliafito CA, et al. Systemic bevacizumab (Avastin) therapy for neovascular age-related macular degeneration: twenty-four-week results of an uncontrolled openlabel clinical study. Ophthalmology 2006;113:2002-2012.

30. Aisenbrey S, Ziemssen F, Volker M, et al. Intravitreal bevacizumab (Avastin) for occult choroidal neovascularization in agerelated macular degeneration. Graefes Arch Clin Exp Ophthalmol 2007:245:941-948.

31. Calvo-Gonzalez C, Reche-Frutos J, Donate-Lopez J, et al. Combined pegaptanib sodium (Macugen) and photodynamic therapy in predominantly classic juxtafoveal choroidal neovascularisation in age related macular degeneration. $\mathrm{Br} \mathrm{J}$ Ophthalmol 2008; 92:74-75.

32. Antoszyk AN, Tuomi L, Chung CY, Singh A. Ranibizumab combined with verteporfin photodynamic therapy in neovascular age-related macular degeneration (FOCUS): year 2 results. Am J Ophthalmol 2008;145:862-874. 